Economic Computation and Economic Cybernetics Studies and Research, Issue 2/2019; Vol. 53

\author{
Student Zhiqiang Liao \\ E-mail: liaozhiqiang_m@163.com \\ Professor Huchang Liao, PhD \\ E-mail: liaohuchang@163.com (Corresponding author) \\ Student Xunjie Gou \\ E-mail: gouxunjie@qq.com \\ Professor Zeshui Xu, PhD \\ E-mail: xuzeshui@ 263.net \\ Business School, Sichuan University, Chengdu, China \\ Professor Edmundas Kazimieras ZAVADSKAS \\ E-mail: edmundas.zavadskas@vgtu.It \\ Institute of Sustainable Construction \\ Vilnius Gediminas Technical University, Vilnius, Lithuania
}

\title{
A HESITANT FUZZY LINGUISTIC CHOQUET INTEGRAL-BASED MULTIMOORA METHOD FOR MULTIPLE CRITERIA DECISION MAKING AND ITS APPLICATION IN TALENT SELECTION
}

Abstract In qualitative multiple criteria decision making (MCDM) process, dealing with complex linguistic evaluations and modeling interaction phenomena among criteria are two important issues. The purpose of this study is to introduce two Choquet integral operators for HFLTSs to tackle interactions among criteria and then implement these operators to a well-known MCDM method, namely, the MULTIMOORA (multi-objective optimization by ratio analysis plus the full multiplicative form). Firstly, we introduce new operations for HFLTSs based on some linguistic scale functions. Then, we define two hesitant fuzzy linguistic Choquet integral operators and propose a novel score function for hesitant fuzzy linguistic elements. Afterwards, we improve the HFL-MULTIMOORA method to handle the hesitant fuzzy linguistic MCDM problems in which the criteria are inter-dependent. Finally, an illustration regarding the human resource development at Sichuan University is shown to verify the applicability and validity of the proposed method.

Keywords: Multiple criteria decision making; Hesitant fuzzy linguistic term set; Score function; Choquet integral; MULTIMOORA.

JEL classification: D81, M12, M51, 015

\section{Introduction}

Multiple criteria decision making (MCDM), taking place widely in many fields such as pattern recognition, finance and military decision, has been studied by many 
Zhiqiang Liao, Huchang Liao, Xunjie Gou, ZeshuiXu, EdmundasKazimierasZavadskas researchers(Ho et al., 2010; Wu and Liao, 2018). Two important processes to solve MCDM problems are: 1) evaluating the performance of each alternative with respect to different criteria, and 2) aggregating the evaluations given by experts and ranking the alternatives(Liao et al., 2019).Two major difficulties for experts to evaluate the performance of alternatives are the lack of accurate information and the limit of their cognition. Instead of single linguistic terms, experts often prefer to express their evaluations on alternatives via complex linguistic expressions, such as "less than important", "at least medium", and "between a little important and very important". In this situation, it is adequate to use the hesitant fuzzy linguistic term set (HFLTS)(Rodriguez et al., 2012), whose elements are ordered subsets of linguistic terms, to indicate the hesitant fuzzy linguistic information. Using the HFLTS, the richness and flexibility of the fuzzy linguistic approach(Zadeh, 1975) would be increased.Liao et al. (2015b)introduced the mathematical definition of the HFLTS and named the element of an HFLTS as the hesitant fuzzy linguistic element (HFLE) for the convenience of calculation.

Regarding to the second issue about aggregating the evaluations given by experts, the MULTIMOORA (multi-objective optimization by ratio analysis plus the full multiplicative form), as a widely used MCDM method, provides a robust ranking method involving three subsystems, such as the ratio system, the reference point approach, and the full multiplicative form(Brauers and Zavadskas, 2011). Due to the good performance compared with other decision-making methods, the MULTIMOORA have been investigated by many scholars from different aspects, such as the fuzzy linguistic MULTIMOORA for group decision making (GDM)(Chen et al., 2018), the double hierarchy hesitant fuzzy linguistic MULTIMOORA (Gou et al., 2017), and the probabilistic linguistic MULTIMOORA with improved Borda rule(Wu et al., 2018). Recently, Liao et al. (2019)investigated the hesitant fuzzy linguistic MULTIMOORA (HFL-MULTIMOORA) based on the score function of HFLEs considering the hesitancy degrees and linguistic scale functions of linguistic terms. Nevertheless, these researches ignored the inter-dependency among criteria. This paper dedicates to filling this research gap. The Choquet integral (Choquet, 1954), which identifies the importance of each criterion by a measure, provides a valid approach to aggregate evaluations over inter-dependent criteria. Sugeno (1974)proposed the concept of fuzzy measure to depict the interactive characteristics among criteria for MCDM problems. Based on the Choquet integral and fuzzy measure, an interval-valued intuitionistic hesitant fuzzy Choquet integral operator was introduced to handle the multiple criteria GDM problems under the interval-valued intuitionistic environment(Joshi and Kumar, 2016). However, as far as we know, there is no paper investigating the hesitant fuzzy linguistic MULTIMOORA method based on Choquet integral.

Considering the defects analyzed above, in this paper, we introduce a Choquet integral-based HFL-MULTIMOORA method in the situation that there are interactions among criteria and interactive connections among multiple decision-makers in the GDM process. Inspired by the work of Liao et al. (2019), a new score function based on the linguistic scale functions of linguistic terms and the 
A Hesitant Fuzzy Linguistic Choquet Integral-based MULTIMOORA Method for Multiple Criteria Decision Making and Its Application in Talent Selection

hesitancy degrees of HFLEs is proposed as a comparison method to obtain the permutation of HFLEs used in the hesitant fuzzy linguistic Choquet integral aggregation operator. It can also be used in the HFL-MULTIMOORA to obtain the utility values of the aggregated HFLEs that are obtained by the proposed hesitant fuzzy linguistic Choquet integral aggregation operators. In addition, the existing operations on HFLEs were mainly based on the addition and multiplication of the subscripts of linguistic terms (Liao et al., 2018). In practice, there is no sense in computing the subscripts of linguistic terms with different scales. In this regard, we propose new operations of HFLEs based on the weights of HFLEs and linguistic scale functions. To reflect the interactions among criteria, we use the fuzzy measure to represent the important information of individual criteria and define two hesitant fuzzy linguistic Choquet integral (HFLCI) aggregation operators in which the additivity condition is replaced by the monotonic property. Then, we extend the HFLCI operators to MULTIMOORA method to solve the MCDM problems with inter-dependent criteria in the hesitant fuzzy linguistic context and obtain the final ranking by the HFL-ORESTE method(Wu and Liao, 2018).

The primary contributions of this paper are summarized as follows:

(1) We propose new operations of HFLTSs based on the weights of HFLEs and the linguistic scale functions of linguistic terms. These operations are more rational than the existing ones.

(2) We propose a score function to obtain the permutation of HFLEs, and utilize the score function to obtain the utility values of alternatives.

(3) We introduce a Choquet integral-based HFL-MULTIMOORA method to handle the situation where criteria are inter-dependent on each other.

(4) We implement the proposed Choquet integral-based HFL-MULTIMOORA method to a numerical example regarding the human resource development at Sichuan University to illustrate the applicability and validity of the proposed method.

The rest of this paper is organized as follows: Section 2 describes the HFLTS, the subsystems of the MULTIMOORA and some concepts of fuzzy measure and Choquet integral. Section 3 proposes new operations of HFLEs. A novel score function of the HFLE and the HFLCI aggregation operators are then introduced. In Section 4, we propose a Choquet integral-based HFL-MULTIMOORA method for MCDM problems based on the proposed HFLCI operators and the score function of HFLEs. An illustrative example regarding the talent section at Sichuan University is presented to show the proposed method and a validity test for this method is provided in Section 5. Conclusions are pointed out in Section 6.

\section{Preliminaries}

In this section, we review the concepts of HFLTS and the main idea of the MULTIMOORA method for MCDM problems. Then, we describe the concepts of the fuzzy measure and Choquet integral.

\subsection{Hesitant fuzzy linguistic term set}

To evaluate qualitative information intuitively and flexibly, Zadeh 
Zhiqiang Liao, Huchang Liao, Xunjie Gou, ZeshuiXu, EdmundasKazimierasZavadskas

(1975)introduced the fuzzy linguistic approach. By extending this approach to hesitant environment, Rodriguez et al. (2012) introduced the concept of HFLTS as an ordered finite subset of the consecutive linguistic terms of a linguistic term set (LTS), which provides a set of linguistic terms to represent the evaluations of decision-makers. Later, Liao etal. (2015b) redefined the HFLTS mathematically as $H_{S}=\left\{<x_{i}, h_{S}\left(x_{i}\right)>\mid x_{i} \in X\right\}$ where

$$
h_{S}(x)=\left\{s_{\phi_{l}}(x) \mid s_{\phi_{l}}(x) \in S, \phi_{l} \in\{-\tau, \cdots, 0, \cdots, \tau\}, l=1,2, \cdots, L(x)\right\}
$$

With $L(x)$ being the number of linguistic terms in $h_{S}(x)$ and $s_{\phi_{l}}(x)$ $(l=1,2, \cdots, L(x))$ being the continuous terms in the LTS $S=\left\{s_{t} \mid t \in\{-\tau, \cdots, 0, \cdots\right.$, $\tau\}\} . h_{S}(x)$ indicatesa set of possible degrees of the linguistic variable $x$ to $S$ and is named the HFLE. For example, let $S=\left\{s_{0}=\right.$ bad, $s_{1}=$ a little bad, $s_{2}=$ medium, $s_{3}=$ a little good,$s_{4}=$ good $\}$ be an LTS. Then, $h_{S}^{1}=\left\{s_{0}=\right.$ bad, $s_{1}=$ a little bad , $s_{2}=$ medium $\}$ and $h_{S}^{2}=\left\{s_{2}=\right.$ medium,$s_{3}=$ alittle good,$s_{4}=$ good $\}$ are two different HFLEs.

As we can see, the HFLEs are not consistent with human thinking and expression. To align with human cognition and make it compatible with the traditional singleton linguistic terms, Rodriguez et al. (2012)proposed a translation function $E_{G_{H}}$ to transform the linguistic expression $l l$, obtained by the context free grammar $G_{H}$, into an HFLE $h_{S}$, where $E_{G_{H}}: l l \rightarrow h_{S}$. Regarding to different types of linguistic expressions, the transformation functions may be in different forms, such as:

(1) $E_{G_{H}}\left(s_{t}\right)=\left\{s_{t} \mid s_{t} \in S\right\}$;

(2) $E_{G_{H}}$ (at most $\left.s_{i}\right)=\left\{s_{t} \mid s_{t} \in S\right.$ and $\left.s_{t} \leq s_{i}\right\}$;

(3) $E_{G_{H}}$ (less than $s_{i}$ ) $=\left\{s_{t} \mid s_{t} \in S\right.$ and $\left.s_{t}<s_{i}\right\}$;

(4) $E_{G_{H}}$ (at least $\left.s_{i}\right)=\left\{s_{t} \mid s_{t} \in S\right.$ and $\left.s_{t} \geq s_{i}\right\}$;

(5) $E_{G_{H}}$ (more than $s_{i}$ ) $=\left\{s_{t} \mid s_{t} \in S\right.$ and $\left.s_{t}>s_{i}\right\}$;

(6) $E_{G_{H}}$ (between $s_{i}$ and $s_{j}$ ) $=\left\{s_{t} \mid s_{t} \in S\right.$ and $\left.s_{i} \leq s_{t} \leq s_{j}\right\}$.

\subsection{The MULTIMOORA method}

The MULTIMOORA method has three subordinate ranking models, including the ratio system, reference point approach and full multiplication form. Each of these three models has shortcomings and advantages. To improve their robustness, Brauers and Zavadskas (2010)introduced the MULTIMOORA to integrate these three models and obtain all the advantages of them and avoid their limitations. To integrate the outcomes of these three subordinate parts, a variety of data fusion techniques can be deployed, such as the dominance theory(Brauers and Zavadskas, 2011), the improved Bordarule(Wu et al., 2018) and the ORESTE method (Liao et 
A Hesitant Fuzzy Linguistic Choquet Integral-based MULTIMOORA Method for Multiple Criteria Decision Making and Its Application in Talent Selection

al., 2019).Recently, Hafezalkotob et al. (2019)conducted an overview of MULTIMOORA by classifying and analyzing the main developments and applications from theoretical and practical points of view.

Consider an MCDM problem with $m$ alternatives $\left\{a_{1}, a_{2}, \cdots, a_{m}\right\}$ and $n$ criteria $\left\{c_{1}, c_{2}, \cdots, c_{n}\right\}$. Let $D=\left(x_{i j}\right)_{n \times m}$ be a general decision matrix, where $x_{i j}$ denotes the performance of alternative $a_{i}$ on criterion $c_{j}$. The weight of criterion $c_{j}$ is supposed to be $\omega_{j}$. The normalization is an important stage of the MULTIMOORA. However, it has been proven that none of the normalization methods are significantly better than others in all cases (Kosarevaet al., 2018). To obtain the ranking of alternatives, the resubordinate rankings can be calculated by:

$$
\begin{aligned}
& U_{1}\left(a_{i}\right)=\sum_{j=1}^{g} \omega_{j} x_{i j}^{N}-\sum_{j=g+1}^{n} \omega_{j} x_{i j}^{N} \\
& U_{2}\left(a_{i}\right)=\max _{j} \omega_{j}\left|r_{j}-x_{i j}^{N}\right| \\
& U_{3}\left(a_{i}\right)=\prod_{j=1}^{g}\left(x_{i j}^{N}\right)^{\omega_{j}} / \prod_{j=g+1}^{n}\left(x_{i j}^{N}\right)^{\omega_{j}}
\end{aligned}
$$

where $c_{j}(j=1,2, \cdots, g)$ are benefit criteria and $c_{j}(j=g+1, \cdots, n)$ are cost criteria, and $r_{j}=\max _{i} x_{i j}^{N}$ if $j=1,2, \cdots, g ; r_{j}=\min _{i} x_{i j}^{N}$ if $j=g+1, g+2, \cdots, n$.

According to the values of $U_{1}\left(a_{i}\right), U_{2}\left(a_{i}\right)$ and $U_{3}\left(a_{i}\right)(i=1,2, \cdots, m)$, we can obtain three subordinate rankings. Then, the final alternative ranking can be derived by integrating the outcomes of these three subordinate parts. For details, please refer to the survey paper (Hafezalkotobet al., 2019).

\subsection{Fuzzy measure and Choquet integral}

Choquet integral provides an approach to define the interaction of criteria and the Choquet integral aggregation operator is regarded as a reasonable method to fuse information over dependent criteria in the MCDM problems. Suppose that we use a fuzzy measure $\mu$ to convey the importance of the subsets of criteria. The set function $\mu: 2^{C} \rightarrow[0,1]$ should satisfy the following properties(Sugeno, 1974):1) $\mu(\phi)=0$;2) $\mu(C)=1$;3) $\mu(A) \geq \mu(B)$. With the separate weights of criteria, we can define the weights of any interaction of criteria. However, it is difficult to determine the fuzzy measure only according to the above properties. Thus, Sugeno(1974)proposed the following $\lambda$-fuzzy measure:

$$
\mu(A \cup B)=\mu(A)+\mu(B)+\lambda \mu(A) \mu(B)
$$

where $A \cap B=\phi$ and $\lambda \in[0,1]$ determines the interaction between criteria. If $\lambda=0$, then the $\lambda$-fuzzy measure is reduced to a simple additive measure. The parameter $\lambda$ can be determined by solving the following equation: 
Zhiqiang Liao, Huchang Liao, Xunjie Gou, ZeshuiXu, EdmundasKazimierasZavadskas

$$
1+\lambda=\prod_{j=1}^{n}\left(1+\lambda \mu\left(x_{j}\right)\right)
$$

Let $\mu$ be a fuzzy measure on $X$, and $f$ be a positive real-valued function on $X$. The discrete Choquet integral of $f$ with respect to $\mu$ is defined as(Grabisch $e t$ al., 2000):

$$
C_{\mu}(f)=\sum_{j=1}^{n}\left[f\left(x_{\sigma(j)}\right)-f\left(x_{\sigma(j+1)}\right)\right] \mu\left(A_{j}\right)
$$

where $\sigma(\cdot)$ is a permutation of $(1,2, \cdots, n)$ such that $0 \leq f\left(x_{\sigma(1)}\right) \leq f\left(x_{\sigma(2)}\right)$ $\leq \cdots \leq f\left(x_{\sigma(n)}\right)$, and $A_{j}=\left\{x_{\sigma(j)}, x_{\sigma(j+1)}, \cdots, x_{\sigma(n)}\right\}$ is a subset of $n-j+1$ largest components in $X$ and $A_{n+1}=\phi$.

3. Hesitant fuzzy linguistic Choquet integral operators based on the score function of HFLEs

In this section, the operations of HFLEs based on the semantics of linguistic terms are addressed. Then, a comprehensive score function of HFLEsis proposed to translate the qualitative HFLEs into numerical values. Afterwards, two HFLCI operators, which use the fuzzy measure to depict the inter-dependency among criteria, are proposed to fuse the evaluation information on inter-dependent criteria in MCDM problems.

\subsection{Operations of HFLEs based on linguistic scale functions}

The operations based on the integer subscripts of linguistic terms have been applied widely in the calculation of linguistic terms(Xu 2006). However, this may lead to some drawbacks. Firstly, the calculation results with respect to the subscripts of linguistic terms may exceed the bounds of LTSs. In addition, it is difficult to compute HFLEs since there area set of linguistic terms in each HFLE. To address this issue, the linguistic scale functions of linguistic terms were investigated by many scholars based on the semantics of the linguistic terms (Liao et al., 2019).

Let $\theta_{\alpha} \in[0,1]$ be a numeric value corresponding to the linguistic term $s_{\alpha}$. Generally, the linguistic scale function $g: s_{\alpha} \rightarrow \theta_{a}$ that translates the linguistic term $s_{\alpha}$ to its corresponding semantic value $\theta_{\alpha}$ is a monotonically increasing function with respect to the degrees of subscripts. That is to say, $g\left(s_{\alpha}\right)>g\left(s_{\beta}\right)$, if $s_{\alpha}>s_{\beta}$. The linguistic scale functions and their inverse functions have three different forms (Liao et al., 2019; Wu and Liao, 2019). For simplicity of the presentation, here we only consider the case that the semantics are uniformly distributed. In this case, we have

$$
\left\{\begin{array}{l}
g\left(s_{\alpha}\right)=(\alpha+\tau) / 2 \tau \\
g^{-1}\left(\theta_{a}\right)=2 \tau \cdot \theta_{a}-\tau
\end{array}\right.
$$

One obvious defect of existing operations is that the sum of results of the scale function may still exceed the domain of the inverse function. For example, if the LTS 
A Hesitant Fuzzy Linguistic Choquet Integral-based MULTIMOORA Method for Multiple Criteria Decision Making and Its Application in Talent Selection

is $S=\left\{s_{-3}=\right.$ very bad, $s_{-2}=$ bad, $s_{-1}=$ little bad, $s_{0}=$ medium, $s_{1}=$ little good, $s_{2}=$ good,$s_{3}=$ very good $\}$, and two HFLEs are $h_{1}=\left\{s_{2}\right\}$ and $h_{2}=\left\{s_{3}\right\}$, then we have $h_{1} \oplus h_{2}=g^{-1}\left(g\left(s_{2}\right)+g\left(s_{3}\right)\right)$. For convenience, suppose that the semantics are uniformly distributed, i.e, the linguistic scale function is given as Eq. (8). Then, we have $g\left(s_{2}\right)+g\left(s_{3}\right)=11 / 6 \notin[0,1]$.Obviously, it is unreasonable. To avoid this defect, we define the addition and multiplication operations on HFLEs as follows:

Theorem 1. Let $h_{S}^{j}(j=1,2, \cdots, n)$ be a collection of HFLEs. The generalized forms of (1) and (2) are:

(1) $\oplus_{j=1}^{n} w_{j} h_{S}^{j}=\bigcup_{s_{\alpha_{l}}^{j} \in h_{S}^{j}}\left\{g^{-1}\left(\sum_{j=1}^{n}\left(w_{j} g\left(s_{\alpha_{l}}^{j}\right)\right)\right)\right\}$,

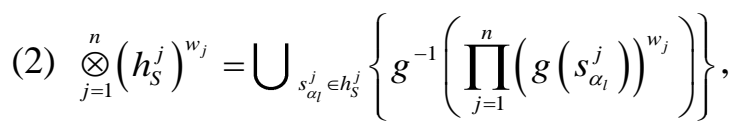

where $w_{j} \in[0,1]$ and $\sum_{j=1}^{n} w_{j}=1$.

For example, let $S=\left\{s_{\alpha} \mid \alpha \in\{-3,-2,-1,0,1,2,3\}\right\} \quad$ be an LTS and the semantics of linguistic terms are uniformly distributed, namely, $g\left(s_{\alpha}\right)=\frac{\alpha+3}{6}$. For two HFLEs $h_{S}^{1}=\left\{s_{-1}, s_{0}, s_{1}\right\}$ and $h_{S}^{2}=\left\{s_{2}, s_{3}\right\}$ with $w_{1}=0.4$ and $w_{2}=0.6$ being the weights of them respectively, we have

$$
\begin{aligned}
& w_{1} h_{S}^{1} \oplus w_{2} h_{S}^{2}=\left\{s_{0.800}, s_{1.400}, s_{1.200}, s_{1.800}, s_{1.600}, s_{2.200}\right\}, \\
& \left(h_{S}^{1}\right)^{w_{1}} \otimes\left(h_{S}^{2}\right)^{w_{2}}=\left\{s_{0.466}, s_{0.866}, s_{1.076}, s_{1.547}, s_{1.573}, s_{2.102}\right\} .
\end{aligned}
$$

We can find that all the linguistic terms in the calculated results are within the bounds of the given LTS.

\subsection{A new score function of the HFLE}

To obtain the permutation of HFLEs, Liao et al. (2015a)introduced a score function of HFLEs based on the average of the integer subscripts of the linguistic terms. Taking into account the unbalanced semantics of an ordered set of linguistic terms, a new score function named the Score-HeDLiSF was then proposed by Liao et al. (2019)based on the hesitancy degree and the linguistic scale functions of the linguistic terms, shown as:

$$
E_{\text {Liao }}\left(h_{S}\right)=\left(1-H D_{\text {Liao }}\left(h_{S}\right)\right) \times \frac{1}{\# h_{S}} \sum_{s_{\alpha} \in h_{S}} g\left(s_{\alpha}\right)
$$

where $\# h_{S}$ denotes the number of elements in $h_{S}, H D_{\text {Liao }}\left(h_{S}\right)=$ $\# h_{S} \ln \left(\# h_{S}\right) /(2 \tau+1) \ln (2 \tau+1)$ is the hesitancy degree of $h_{S}$, and $g\left(s_{\alpha}\right)$ is the 
Zhiqiang Liao, Huchang Liao, Xunjie Gou, ZeshuiXu, EdmundasKazimierasZavadskas linguistic scale function.

This semantic-based score function takes into account the linguistic scale function of the LTS and the hesitancy degree of HFLEs, but the hesitancy degree function, a monotonically increasing concave function, is difficult to be determined. In addition, this hesitancy degree does not consider the deviations between linguistic terms. Thus, it is necessary to put forward a new score function of HFLEs to overcome that defect. Firstly, we propose a new hesitancy degree function of an HFLE $h_{S}$ based on the deviation of linguistic terms:

$$
H D\left(h_{S}\right)=\sqrt{\frac{1}{\# h_{S}} \sum_{s_{\alpha} \in h_{S}}\left(g\left(s_{\alpha}\right)-\tilde{g}\left(s_{\alpha}\right)\right)^{2}}
$$

where $\tilde{g}\left(s_{\alpha}\right)=\frac{1}{\# h_{S}} \sum_{s_{\alpha} \in h_{S}} g\left(s_{\alpha}\right)$.

From the above definition, we can see that the hesitancy degree function is similar to the variance in statistics. It is worth noting that $H D\left(h_{S}\right)$ represents the dispersion degree of the semantics of linguistic terms.In what follows, we define a new score function of an HFLE $h_{s}$ as:

$$
E\left(h_{S}\right)=\left(\frac{1}{\# h_{S}} \sum_{s_{\alpha} \in h_{S}} g\left(s_{\alpha}\right)^{\left(1-H D\left(h_{S}\right)\right)}\right)^{1 /\left(1-H D\left(h_{S}\right)\right)}
$$

When using the hesitancy degree to construct the score function of an HFLE, we see that $1-H D\left(h_{S}\right)$ can be regarded as a type of nominal weight associated with the HFLE $h_{S}$.For any two HFLEs, if $E\left(h_{S}^{1}\right)>E\left(h_{S}^{1}\right)$, then $h_{S}^{1}>h_{S}^{2}$; if $E\left(h_{S}^{1}\right)=E\left(h_{S}^{1}\right)$, then $h_{S}^{1}=h_{S}^{2}$. For example, for two HFLEs $h_{S}^{1}=\left\{s_{-1}, s_{0}, s_{1}\right\}$ and $h_{S}^{2}=\left\{s_{2}, s_{3}\right\}$, by Eq. (11), the scores of them can be calculated as $E\left(h_{S}^{1}\right)=0.497$ and $E\left(h_{S}^{2}\right)=0.916$. Then, we have $E\left(h_{S}^{1}\right)<E\left(h_{S}^{2}\right)$. Thus, there is $h_{S}^{1}<h_{S}^{2}$.

\subsection{Hesitant fuzzy linguistic Choquet integral operator}

Considering the interaction of criteria and the preferences of decision-makers, Joshi and Kumar (2016) proposed the interval-valued intuitionistic hesitant fuzzy Choquet integral. Based on the HFLTS and Choquet integral, in this section, we define two HFLCI operators to take into account the inter-dependency between criteria, where the individual criterion importance is represented by a fuzzy measure.

Let $\mu$ be a fuzzy measure on $C=\left\{c_{1}, c_{2}, \cdots, c_{j}, \cdots, c_{n}\right\}$, and $h_{S}^{j}$ $(j=1,2, \cdots, n)$ be a collection of HFLEs on the LTS $S$. Then, a hesitant fuzzy linguistic Choquet integral average operator (HFLCIA) and a hesitant fuzzy linguistic Choquet integral geometric operator (HFLCIG) are defined as:

$$
\operatorname{HFLCIA}\left(h_{S}^{1}, h_{S}^{2}, \ldots, h_{S}^{n}\right)=\bigoplus_{j=1}^{n}\left(h_{S}^{\sigma(j)}\right)\left(\mu\left(A_{j}\right)-\mu\left(A_{j+1}\right)\right)
$$


A Hesitant Fuzzy Linguistic Choquet Integral-based MULTIMOORA Method for Multiple Criteria Decision Making and Its Application in Talent Selection

$$
\operatorname{HFLCIG}\left(h_{S}^{1}, h_{S}^{2}, \ldots, h_{S}^{n}\right)=\bigotimes_{i=1}^{n}\left(h_{S}^{\sigma(i)}\right)^{\left(\mu\left(A_{j}\right)-\mu\left(A_{j+1}\right)\right)}
$$

where $\sigma(j) \quad(j=1,2, \cdots, n)$ is a permutation of $(1,2, \cdots, n)$ such that $h_{S}^{\sigma(1)} \leq h_{S}^{\sigma(2)} \leq \cdots \leq h_{S}^{\sigma(n)}, A_{j}=\left\{h_{S}^{\sigma(i)}, h_{S}^{\sigma(i+1)}, \cdots, h_{S}^{\sigma(n)}\right\}$ and $h_{S}^{\sigma(n+1)}=\varnothing$.

Theorem 2. The HFLCIA and HFLCIG operators are calculated as:

$$
\begin{aligned}
& \operatorname{HFLCIA}\left(h_{S}^{1}, h_{S}^{2}, \ldots, h_{S}^{n}\right)=\bigoplus_{j=1}^{n}\left(h_{S}^{\sigma(j)}\right)\left(\mu\left(A_{j}\right)-\mu\left(A_{j+1}\right)\right) \\
& =\bigcup_{s_{\alpha_{l}}^{\sigma(j)} \in h_{S}^{\sigma(j)}}\left\{g^{-1}\left(\sum_{j=1}^{n}\left(\left(\mu\left(A_{j}\right)-\mu\left(A_{j+1}\right)\right) g\left(s_{\alpha_{l}}^{\sigma(j)}\right)\right)\right)\right\} \\
& \operatorname{HFLCIG}\left(h_{S}^{1}, h_{S}^{2}, \ldots, h_{S}^{n}\right)=\bigotimes_{i=1}^{n}\left(h_{S}^{\sigma(i)}\right)^{\left(\mu\left(A_{j}\right)-\mu\left(A_{j+1}\right)\right)} \\
& =\bigcup_{s_{\alpha_{l}}^{\sigma(j)} \in h_{S}^{\sigma(j)}}\left\{g^{-1}\left(\prod_{j=1}^{n}\left(g\left(s_{\alpha_{l}}^{\sigma(j)}\right)\right)^{\left(\mu\left(A_{j}\right)-\mu\left(A_{j+1}\right)\right)}\right)\right\}
\end{aligned}
$$

Proof. Theorem 2 can be proven easily by mathematical induction. Here we omit the proof here due to the limited pages of the paper.

We can get some important properties of the HFLCI aggregation operators.

Theorem 3. The HFLCIA operator satisfies: 1) $h_{S}{ }^{-} \leq \operatorname{HFLCIA}\left(h_{S}^{1}, h_{S}^{2}, \cdots, h_{S}^{n}\right)$ $\leq h_{S}{ }^{+}$, where $h_{S}{ }^{-}=\min _{j=1}^{n}\left\{h_{S}^{j}\right\}$ and $\left.h_{S}{ }^{+}=\max _{j=1}^{n}\left\{h_{S}^{j}\right\} ; 2\right)$ If $h_{S}^{j} \leq \tilde{h}_{S}^{j}$, then, $\operatorname{HFLCIA}\left(h_{S}^{1}, h_{S}^{2}, \cdots, h_{S}^{n}\right) \leq \operatorname{HFLCIA}\left(\tilde{h}_{S}^{1}, \tilde{h}_{S}^{2}, \cdots, \tilde{h}_{S}^{n}\right)$.

The proof is easy and thus we omit it here. In addition, it is not difficult to prove that the HFLCIG operator also satisfies the above two properties. In the following of this paper, we always take the HFLCIA operator as an illustration.

4. A Choquet integral-based HFL-MULTIMOORAmethod for MCDM

In this section, we propose a Choquet integral-based HFL-MULTIMOORA method for MCDM problems. The algorithm of the Choquet integral-based HFL-MULTIMOORA method is given for the facility of application.

\subsection{Representing the importance of criteria by the fuzzy measure}

For an MCDM problem, we use the HFLE $h_{S}^{i j}$ to denote the performance of alternative $a_{i}$ regarding criterion $c_{j}$. Under the hesitant fuzzy linguistic environment, decision-makers provide sets of linguistic terms or linguistic expressions instead of real values or single linguistic terms to express their assessments. To aggregate the assessments over the criteria, we need to determine the criteria weights first for solving the MCDM problem.

Measure is an important tool to represent the weight of a subset of criteria. The 
Zhiqiang Liao, Huchang Liao, Xunjie Gou, ZeshuiXu, EdmundasKazimierasZavadskas prototypical situation is that, for each criterion $c_{j}$, there is a measure $\mu\left(c_{j}\right)$, which identifies the importance of the criterion $c_{j}$. The classical measure is supposed to be additive. That is to say, $\mu(C)=\sum_{c_{j} \in C} \mu\left(c_{j}\right)$. In this situation, $\mu(C)=1$ if $C$ is the full set of criteria. Thus, $\sum_{j=1}^{n} \mu\left(c_{j}\right)=1$.However, the practical situation may be more complicated than the prototypical one. Using a non-additive measure to represent the weights of criteria is more appropriate than the basic additive measure especially when the relationship between criteria is inter-dependent. The fuzzy measure $\mu$ can provide a possibility such that $\sum_{k=1}^{q} \mu\left(c_{k}\right) \neq 1$. Besides, the fuzzy measure allows other situations such as $\mu\left(c_{1}, c_{2}\right) \neq \mu\left(c_{1}\right)+\mu\left(c_{2}\right)$. For example, the weight measures of criteria $\left\{c_{1}, c_{2}, c_{3}\right\}$ could be $\mu\left(c_{1}\right)=0.3, \mu\left(c_{2}\right)=0.6$ and $\mu\left(c_{3}\right)=0.5$, respectively. This is to say, we allow that $\mu\left(c_{1}\right)+\mu\left(c_{2}\right)+\mu\left(c_{3}\right) \neq 1$. If we have $\mu\left(c_{1}, c_{2}\right)=0.8$, then we also allow that $\mu\left(c_{1}, c_{2}\right) \neq \mu\left(c_{1}\right)+\mu\left(c_{2}\right)$.

4.2. An HFL-MULTIMOORA method integrated with the Choquet integral and the score function of HFLEs

The MULTIMOORA method is a power technique for solving MCDM problems(Brauers and Zavadskas, 2011). Based on the new score function of HFLEs (Eq. (11)), this section dedicates to giving a Choquet integral-based HFL-MULTIMOORA method to solve the hesitant fuzzy linguistic MCDM problems in which the criteria are interdependent.

Since the original HFL-MULTIMOORA method (Liao et al., 2019)is almost invalid if there exists inter-dependency or interaction among criteria, we use the HFLCI aggregation operators to identify the relationships between criteria. After the aggregation results are obtained, we then use the score function given as Eq. (11) to obtain the subordinate utility values. The three extended aggregation models in the Choquet integral-based HFL-MULTIMOORA method can be given as follows:

(1) The HFLCI-based ratio system (HFLCIRS)

With the HFLCIA operator, the HFLEs $h_{S}^{i j}(j=1,2, \cdots, n)$ of alternative $a_{i}$ on criteria $c_{j}(j=1,2, \cdots, n)$ can be aggregated. Then, the utility of the ratio system based on the HFLCIA operator can be calculated by:

$$
U_{1}\left(a_{i}\right)=E\left(\operatorname{HFLCIA}\left(h_{S}^{1}, h_{S}^{2}, \cdots, h_{S}^{g}\right)\right)-E\left(\operatorname{HFLCIA}\left(h_{S}^{g+1}, h_{S}^{g+2}, \cdots, h_{S}^{n}\right)\right)
$$

where $E\left(h_{S}\right)$ is the score function proposed in Eq. (11), $c_{j}(j=1,2, \cdots, g)$ are benefit criteria and $c_{j}(j=g+1, \cdots, n)$ are cost criteria. After the utility values of all alternatives are calculated, we can obtain the ranking of alternative regarding the Ratio System in descending order of $U_{1}\left(a_{i}\right) \quad(i=1,2, \cdots, m)$, shown as: 
A Hesitant Fuzzy Linguistic Choquet Integral-based MULTIMOORA Method for Multiple Criteria Decision Making and Its Application in Talent Selection

$R_{1}=\left\{r_{1}\left(a_{1}\right), r_{1}\left(a_{2}\right), \cdots, r_{1}\left(a_{m}\right)\right\}$.

(2) The HFLCI-based reference point system (HFLCIRPS)

Considering the worst performance of $a_{i}$ with respect to each criterion $c_{j}$, we can get the utility of alternative $a_{i}$ in the reference point system as:

$$
U_{2}\left(a_{i}\right)=\max _{\sigma(j)}\left|r_{\sigma(j)}-E\left(h_{S}^{i \sigma(j)}\right)\right|\left(\mu\left(A_{j}(i)\right)-\mu\left(A_{j+1}(i)\right)\right)
$$

where $r_{\sigma(j)}=\max _{\sigma(j)} E\left(h_{S}^{i \sigma(j)}\right)$ if $c_{j}$ is a beneficial criterion and $r_{\sigma(j)}=\min _{\sigma(j)} E\left(h_{S}^{i \sigma(j)}\right)$ if $c_{j}$ is a cost criterion. The ranking of alternative regarding the reference point system is obtained in ascending order of $U_{2}\left(a_{i}\right)(i=1,2, \cdots, m)$, shown as: $R_{2}=\left\{r_{2}\left(a_{1}\right), r_{2}\left(a_{2}\right), \cdots, r_{2}\left(a_{m}\right)\right\}$.

(3) The HFLCI-based full multiplicative form (HFLCIFMF)

To obtain the third subordinate utilities of the alternatives, we use the HFLCIG operator to aggregate the evaluations of expert:

$$
U_{3}\left(a_{i}\right)=E\left(\operatorname{HFLCIG}\left(h_{S}^{1}, h_{S}^{2}, \cdots, h_{S}^{g}\right)\right) / E\left(\operatorname{HFLCIG}\left(h_{S}^{g+1}, h_{S}^{g+2}, \cdots, h_{S}^{n}\right)\right)
$$

Then, we can derive the third ranking of alternatives in descending order of $U_{3}\left(a_{i}\right)$ $(i=1,2, \cdots, m)$, and the ranks of alternatives are: $R_{3}=\left\{r_{3}\left(a_{1}\right), r_{3}\left(a_{2}\right), \cdots, r_{3}\left(a_{m}\right)\right\}$.

After obtaining the three subordinate ranks, we need to aggregate these ranks and get the final ranking of alternatives. The ORESTE(Wu and Liao, 2018) can be used to do so. It is based on a decision matrix $D(R)$ composed by the three subordinate ranks of alternatives, which is in the form as follows:

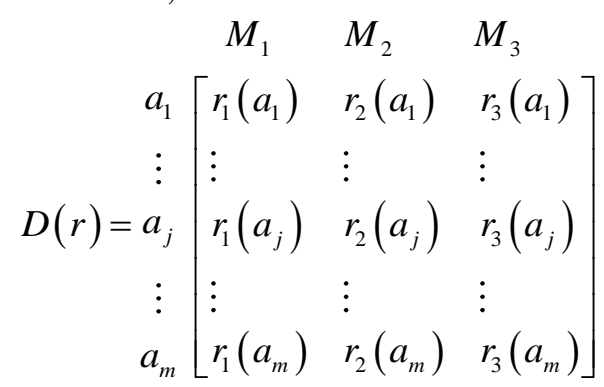

Let $r_{b}\left(a_{i}\right)$ and $r\left(M_{b}\right)$ be the ranks of alternatives and subordinate models, respectively. Note that here we use the Besson's mean ranks(For more details, please refer to $\mathrm{Wu}$ and Liao(2018)) to obtain the global ranking of alternatives. We can compute the Besson's mean rankings $R_{b}\left(a_{i}\right)$ for alternative $a_{i}$ under model $M_{b}$ by:

$$
R_{b}\left(a_{i}\right)=\sqrt{0.5\left(r\left(M_{b}\right)\right)^{2}+0.5\left(r_{b}\left(a_{i}\right)\right)^{2}}
$$


Zhiqiang Liao, Huchang Liao, Xunjie Gou, ZeshuiXu, EdmundasKazimierasZavadskas

Then, we can obtain the global preference score of alternative $a_{i}$ by:

$$
R\left(a_{i}\right)=R_{1}\left(a_{i}\right)+R_{2}\left(a_{i}\right)+R_{3}\left(a_{i}\right)
$$

According to the ascending order of $R\left(a_{i}\right)$, the global ranking of all alternatives can be obtained.

4.3. Procedure of the Choquet integral-based HFL-MULTIMOORA method

To aid the process of solving the qualitative MCDM problems with inter-dependent criteria, below we present the procedure of the proposed method.

Step 1. (Information collection) Determine the alternatives $\left\{a_{1}, \cdots, a_{i}, \cdots, a_{m}\right\}$ and criteria $\left\{c_{1}, \cdots, c_{j}, \cdots, c_{n}\right\}$, and then collect the linguistic evaluations from the expert and establish the decision-making matrices.

Step 2. Divide criteria into two benefit criteria and cost criteria, and then determine the permutation of the preference values for each group according to the score of each HFLE calculated by Eq. (11).

Step 3. Calculate the parameter $\lambda$ for the $\lambda$-fuzzy measure by Eq. (6). According to the importance degree of individual criterion determined by the decision-maker, the fuzzy measures of the criteria can be confirmed by Eq. (5).

Step 4. Utilize the HFLCIA operator shown as Eq. (12)or the HFLCIG operator shown as Eq. (13)to aggregate all HFLEs of each alternative.

Step 5. Use the Choquet integral-based HFL-MULTIMOORA method to calculate the three subordinate utility values $U_{1}\left(a_{i}\right)$ (by Eq. (16)), $U_{2}\left(a_{i}\right)$ (by Eq. (17)) and $U_{3}\left(a_{i}\right)$ (by Eq. (18)). According to the calculated results, we can rank the alternatives and obtain three subordinate ranks $R_{1}\left(a_{i}\right), R_{2}\left(a_{i}\right)$ and $R_{3}\left(a_{i}\right)$.

Step 6. Calculate the global ranking of alternatives by Eqs. (20).

The main advantages of the Choquet integral-based HFL-MULTIMOORA method can be summarized below:

1) We can aggregate the evaluations on the criteria whose important weights do not need to satisfy the additive property. If there are some inter-dependency among the criteria, the method still works.

2) The method takes into account the semantics of different linguistic terms by introducing the linguistic scale function. In addition, the proposed score function of HFLEs is implemented in the method, which can overcome the limitation of the existing score functions (Liao et al., 2019).

3) Compared with the individual methods of the Choquet integral-based HFL-MULTIMOORA method, the integration of these three subordinate ranks can produce a more robust result. In analogous, it also avoids the defects of the existing MCMD methods similar to the three subordinate models.

5. An illustrative example: selecting global talents for a university

In this section, we solve a practical example concerning the selection and recruitment problem of human resource development at a university by the proposed 
A Hesitant Fuzzy Linguistic Choquet Integral-based MULTIMOORA Method for Multiple Criteria Decision Making and Its Application in Talent Selection

Choquet integral-based HFL-MULTIMOORA method. The validity of this method is further demonstrated by a validity test.

\subsection{Case description}

SCU, one of China's top universities, plays an increasingly important role in promoting both national and regional higher education development, and is constantly building its capacities of research and education. The SCU has been included in the "Double First-Class" university program, a significant plan of central Chinese government to enhance the level of China's higher education. Recently, the SCU has proposed some strategies to improve its comprehensive competitiveness, particularly in selecting and promoting the excellent young talents. Selecting an appropriate talent is a very important step to enhance the core competitiveness of an institution. A widespread approach to select talents for universities is to recruit scholars whose names have been listed in various national talent programs, including but not limited to Chang Jiang Scholar Program, Young Overseas High-level Talents Introduction Plan and the National Science Fund for Distinguished Young Scholars (Jiang, 2018). However, because of the limited names of these lists, only a few scholars can be selected in these talent programs and get funding on research. Other researchers without adequate research funding, are struggling to do their research. Due to the lack of resources to carry on their research, many talents have not achieved their full potential in research. In this paper, we aim to find a suitable evaluation system for the SCU to select global talents.

Suppose that the SCU decides to select an appropriate talent from four candidates: Zhou $\left(a_{1}\right), \mathrm{Wu}\left(a_{2}\right)$, Zheng $\left(a_{3}\right)$ and Wang $\left(a_{4}\right)$. The evaluation criteria are education background ( $c_{1}$, benefit), research capacity ( $c_{2}$, benefit), social contribution $\left(c_{3}\right.$, benefit) and expected salary $\left(c_{4}\right.$, cost). To solve the problem, the university construct a committee to evaluate these candidates. The committee furnishes the complex cognitive linguistic information in linguistic expressions, which are further translated to HFLEs. The LTS used to judge the candidates under the first three criteria is predetermined as $S=\left\{s_{-3}=\operatorname{verybad}(v b)\right.$, $s_{-2}=\operatorname{bad}(b), s_{-1}=$ a little bad $(A L B), \quad s_{0}=\operatorname{medium}(M), \quad s_{1}=$ a little $\operatorname{good}(A L G)$, $\left.s_{2}=\operatorname{good}(G), s_{3}=\operatorname{very} \operatorname{good}(V G)\right\}$ and the LTS with respect to the fourth criterion is $S=\left\{s_{-3}=\operatorname{very} \operatorname{low}(V L), s_{-2}=\operatorname{low}(L), s_{-1}=\right.$ a little low $(A L L), s_{0}=\operatorname{medium}(M)$, $s_{1}=$ a little high $\left.(A L H), s_{2}=\operatorname{high}(H), s_{3}=\operatorname{very} \operatorname{high}(V H)\right\}$. The committee was asked to evaluate each candidate under with respect to the four afore mentioned criteria by flexible linguistic expressions. Then, we translated these initial linguistic expressions into HFLEs. Table 1 shows the linguistic evaluations of the committee. 
Zhiqiang Liao, Huchang Liao, Xunjie Gou, ZeshuiXu, EdmundasKazimierasZavadskas

Table 1. The evaluation matrix regarding the four candidates

\begin{tabular}{lllll}
\hline Candidate & $c_{1}$ & $c_{2}$ & $c_{3}$ & $c_{4}$ \\
\hline$a_{1}$ & More than G & More than ALG & G & Between ALL and M \\
$a_{2}$ & ALG & G & More than ALG & Between M and H \\
$a_{3}$ & VG & ALG or G & Between M and G & At least H \\
$a_{4}$ & G & ALG & ALG or G & H or VH \\
\hline
\end{tabular}

5.2. Solve the case by the proposed method

In the following section, we illustrate how to use the proposed method to select the optimal candidate for SCU.

Step 1. The hesitant fuzzy linguistic decision matrix is obtained as:

$$
D=\left[\begin{array}{cccc}
\left\{s_{2}, s_{3}\right\} & \left\{s_{1}, s_{2}, s_{3}\right\} & \left\{s_{2}\right\} & \left\{s_{-1}, s_{0}\right\} \\
\left\{s_{1}\right\} & \left\{s_{2}\right\} & \left\{s_{1}, s_{2}, s_{3}\right\} & \left\{s_{0}, s_{1}, s_{2}\right\} \\
\left\{s_{3}\right\} & \left\{s_{1}, s_{2}\right\} & \left\{s_{0}, s_{1}, s_{2}\right\} & \left\{s_{2}, s_{3}\right\} \\
\left\{s_{2}\right\} & \left\{s_{0}, s_{1}, s_{2}\right\} & \left\{s_{1}, s_{2}\right\} & \left\{s_{2}, s_{3}\right\}
\end{array}\right]
$$

Step 2. We determine the permutation of the preference under the first three benefit criteria according to the score function shown as Eq. (13) and obtain $E\left(h_{S}^{11}\right)=0.916 \quad, \quad E\left(h_{S}^{21}\right)=0.832 \quad, \quad E\left(h_{S}^{31}\right)=0.833 \quad$.Then, we have $E\left(h_{S}^{21}\right)<E\left(h_{S}^{31}\right)<E\left(h_{S}^{11}\right)$ and $h_{S}^{\sigma(1) 1}=h_{S}^{21}, h_{S}^{\sigma(2) 1}=h_{S}^{31}, h_{S}^{\sigma(3) 1}=h_{S}^{11}$.

Step 3. Suppose that the fuzzy measure of the four criteria are represented as: $\mu\left(c_{1}\right)=0.4, \mu\left(c_{2}\right)=0.3, \mu\left(c_{3}\right)=0.3, \mu\left(c_{4}\right)=0.2$. We obtain $\lambda=-0.409$, and $\mu\left(c_{1}, c_{2}\right)=0.651, \mu\left(c_{1}, c_{3}\right)=0.651, \mu\left(c_{2}, c_{3}\right)=0.563, \mu\left(c_{1}, c_{2}, c_{3}\right)=0.871$, $\mu\left(c_{1}, c_{2}, c_{3}, c_{4}\right)=1$.Thus, $\mu\left(A_{\sigma(1)}\right)-\mu\left(A_{\sigma(2)}\right)=0.22, \mu\left(A_{\sigma(2)}\right)-\mu\left(A_{\sigma(3)}\right)=0.251$, $\mu\left(A_{\sigma(3)}\right)-\mu\left(A_{\sigma(4)}\right)=0.4$.

Step 4. Suppose that the semantics of the linguistic terms are uniformly distributed. Utilize the aggregation operators to aggregate $h_{S}^{i j}(j=1,2,3,4)$. Here we omit the calculation results.

Step 5. According to the three aggregation models, the individual utility values of each candidate can be calculated. The results are listed in Table 2.

Table 2. The subordinate utility values and ranks of the candidates

\begin{tabular}{ccccccc}
\hline \multirow{2}{*}{ Candidate } & HFLCIRS & \multicolumn{3}{c}{ HFLCIRPA } & \multicolumn{3}{c}{ HFLCIFMF } \\
\cline { 2 - 3 } \cline { 5 - 6 } & $U_{1}$ & $R_{1}$ & $U_{2}$ & $R_{2}$ & $U_{3}$ & $R_{3}$ \\
\hline$a_{1}$ & 0.705 & 1 & 0.054 & 1 & 0.991 & 1 \\
$a_{2}$ & 0.589 & 3 & 0.086 & 2 & 0.839 & 3 \\
$a_{3}$ & 0.617 & 2 & 0.118 & 3.5 & 0.857 & 2 \\
$a_{4}$ & 0.550 & 4 & 0.118 & 3.5 & 0.797 & 4 \\
\hline
\end{tabular}


A Hesitant Fuzzy Linguistic Choquet Integral-based MULTIMOORA Method for Multiple Criteria Decision Making and Its Application in Talent Selection

Step 6. Suppose that the HFLCIRS model is more important than the HFLCIRPA model, and the HFLCIRPA model is more important than the HFLCIFMF model. Thus, $r\left(M_{1}\right)=1, r\left(M_{2}\right)=2$ and $r\left(M_{3}\right)=3$. By Eq. (20),the global score values of the candidates can be calculated. The results are shown in Table 3:

Table 3. The global score values derived from the ORESTE method

\begin{tabular}{lllll}
\hline Candidate & $M_{1}$ & $M_{2}$ & $M_{3}$ & $r\left(a_{i}\right)$ \\
\hline$a_{1}$ & 1 & 1.581 & 2.236 & 9 \\
$a_{2}$ & 2.236 & 2 & 3 & 20.5 \\
$a_{3}$ & 1.581 & 2.850 & 2.550 & 18 \\
$a_{4}$ & 2.916 & 2.850 & 3.536 & 30.5 \\
\hline
\end{tabular}

Then, the final ranking of the candidates can be obtained as $a_{1}>a_{3}>a_{2}>a_{4}$. Thus, Zhou is the most desirable candidate for SCU.

\subsection{Validity test of the proposed method}

The rank reversal phenomenon is a significant weakness in the MCDM problems when new decision information is added to the decision space (Keshavarz-Ghorabaeeet al., 2018).To evaluate the validity of the proposed method, we use the test criteria introduced by Wang and Triantaphyllou (2008) to show the rationality of the proposed method.

In Table 4, a new linguistic evaluation matrix, obtained by replacing candidate $a_{3}$ (Zheng, non-optimal one) by another candidate $a_{3}^{\prime}$ (Zhang, worse one), is used. Then, according to the Test criterion 1, the indication of the best candidate should not change by the same MCDM method. The calculation results in this case are shown in Table 5 and the global ranking of the candidates can be deduced as: $a_{1}>a_{2}>a_{4}>a_{3}^{\prime}$, so Zhou is still the most desirable one. Thus, the method successfully passes the validity test on the Test Criterion 1 .

Table 4. The modified evaluation matrix regarding the four candidates

\begin{tabular}{lllll}
\hline Candidates & $c_{1}$ & $c_{2}$ & $c_{3}$ & $c_{4}$ \\
\hline$a_{1}$ & More than G & More than ALG & $\mathrm{G}$ & Between L and M \\
$a_{2}$ & ALG & $\mathrm{G}$ & More than ALG & Between M and H \\
$a_{3}^{\prime}$ & $\mathrm{G}$ & ALG or G & Between M and G & VH \\
$a_{4}$ & $\mathrm{G}$ & Between M and G & Between ALG and G & Between H and \\
\hline
\end{tabular}

Table 5. The subordinate utility values and ranks for the modified problem

\begin{tabular}{lllllll}
\hline \multirow{2}{*}{ Candidates } & \multicolumn{2}{l}{ HFLCIRS } & & \multicolumn{2}{l}{ HFLCIRPA } & \multicolumn{2}{l}{ HFLCIFMF } \\
\cline { 2 - 5 } \cline { 6 - 8 } & $U_{1}^{\prime}$ & $R_{1}^{\prime}$ & $U_{2}^{\prime}$ & $R_{2}^{\prime}$ & $U_{3}^{\prime}$ & $R_{3}^{\prime}$ \\
\hline$a_{1}$ & 0.705 & 1 & 0.054 & 1 & 0.991 & 1 \\
\hline
\end{tabular}


Zhiqiang Liao, Huchang Liao, Xunjie Gou, ZeshuiXu, EdmundasKazimierasZavadskas

\begin{tabular}{lllllll}
\hline$a_{2}$ & 0.589 & 2 & 0.086 & 2 & 0.839 & 2 \\
$a_{3}^{\prime}$ & 0.539 & 4 & 0.129 & 4 & 0.787 & 4 \\
$a_{4}$ & 0.550 & 3 & 0.118 & 3 & 0.797 & 3 \\
\hline
\end{tabular}

Suppose that the original decision-making problem is decomposed into two smaller MCDM problems including $\left\{a_{1}, a_{3}, a_{4}\right\}$ and $\left\{a_{1}, a_{2}, a_{3}\right\}$ without changing the criteria and their corresponding weights. Using the Choquet integral-based HFL-MULTIMOORA method, the rankings of the candidates in these two smaller problems are calculated as $a_{1}>a_{2}>a_{4}$ and $a_{1}>a_{3}>a_{2}$. Combining the rankings of candidates of the two problems together, we can obtain the final ranking as $a_{1}>a_{3}>a_{2}>a_{4}$, which is identical to the ranking derived by the original MCDM problem and satisfies the property of transitivity. Hence, the Choquet integral-based HFL-MULTIMOORA method successfully passes the validity test under the Test Criterion 2 and Test Criterion 3. In this sense, the Choquet integral-based HFL-MULTIMOORA method is reasonable for solving MCDM problems, especially when the criteria are inter-dependent.

6. Conclusions and future research directions

This study developed a Choquet integral-based HFL-MULTIMOORA method to deal with the hesitant fuzzy linguistic MCDM problems in the situation that the criteria are not independent. We improved the HFL-MULTIMOORA method by Choquet integral and linguistic scale functions. The HFLCIA and HFLCIG aggregation operators computed by the Choquet integral were proposed. The results of the aggregation operator are still HFLEs, which avoids the loss of information to some extent. In the HFL-MULTIMOORA method, we used three utility models to capture three subordinate rankings of alternatives, which guarantees the robustness of the final ranking results. To show the effectiveness of the proposed method, an illustration of SCU human resource development was presented and a validity test based on the illustration was also implemented.

In the future, it is necessary to investigate the multiple criteria group decision-making problems considering the inter-dependency of criteria and the interaction of decision-makers under the hesitant fuzzy linguistic context. In addition, it would be interesting to use the proposed operations and aggregation operators in other decision-making methods, such as the HFL-VIKOR method (Liao et al., 2015a). Furthermore, exploring more generalized operations of HFLEs rather than the operations with the weights of HFLEs would be also interesting.

\section{ACKNOWLEDGMENTS}

The work was supported by the National Natural Science Foundation of China (71771156).

\section{REFERENCES}

[1] Brauers, W.K.M. and Zavadskas, E.K. (2010), Project Management by MULTIMOORA as an Instrument for Transition Economies. Technological and Economic Development of Economy, 16, 5-24; 
A Hesitant Fuzzy Linguistic Choquet Integral-based MULTIMOORA Method for Multiple Criteria Decision Making and Its Application in Talent Selection

[2] Brauers, W.K.M. and Zavadskas, E.K. (2011), Multimoora Optimization Used to Decide on a Bank Loan to Buy Property. Technological and Economic Development of Economy, 17, 174-188;

[3] Brauers, W.K.M., Kildiene, S., Zavadskas, E.K., Kaklauskas, A. (2013), The Construction Sector in Twenty European Countries During the Recession 2008-2009-Country Ranking by MULTIMOORA. International Journal of Strategic Property Management, 17, 58-78;

[4] Chen, X., Zhao, L., Liang, H. (2018), A Novel Multi-Attribute Group Decision-Making Method Based on the MULTIMOORA with Linguistic Evaluations. Soft Computing, 22, 5347-5361;

[5] Choquet, G. (1954), Theory of Capacities. Annales de l'institut Fourier, 5, 131-295;

[6] Gou, X.J., Liao, H.C., Xu, Z.S., Herrera, F. (2017), Double Hierarchy Hesitant Fuzzy Linguistic Term Set and MULTIMOORA Method: A Case of Study to Evaluate the Implementation Status of Haze Controlling Measures. Information Fusion, 38, 22-34;

[7] Grabisch, M., Murofushi, T., Sugeno, M. (2000), Fuzzy Measures and Integrals-Theory and Applications. Physica Verlag, 40;

[8] Hafezalkotob, A., Hafezalkotob, A., Liao, H.C., Herrera, F. (2019), An Overview of MULTIMOORA for Multi-Criteria Decision-Making: Theory, Developments, Applications, and Challenges. Information Fusion, 51, 145-177;

[9] Ho, W., Xu, X.W., Dey, P.K. (2010), Multi-Criteria Decision Making Approaches for Supplier Evaluation and Selection: A Literature Review. European Journal of Operational Research, 202, 16-24;

[10] Jiang, J. (2018), Competition for Talent and Unequal Development of Higher Education: Evidence from Chang Jiang Scholars Programme. Massification of Higher Education in Asia, Springer, Singapore, 21-37;

[11] Joshi, D., Kumar, S. (2016), Interval-Valued Intuitionistic Hesitant Fuzzy Choquet Integral Based TOPSIS Method for Multi-Criteria Group Decision Making. European Journal of Operational Research, 248, 183-191;

[12] Keshavarz-Ghorabaee, M., Amiri, M., Zavadskas, E.K., Turskis, Z., Antucheviciene, J. (2018), A Comparative Analysis of the Rank Reversal Phenomenon in the EDAS and TOPSIS Methods; Economic Computation and Economic Cybernetics Studies and Research, 52, 121-134;

[13] Kosareva, N., Krylovas, A., Zavadskas, E.K. (2018), Statistical Analysis of MCDM Data Normalization Methods Using Monte Carlo Approach. The Case of Ternary Estimates Matrix. Economic Computation and Economic Cybernetics Studies and Research, 52,159-175;

[14] Liao, H.C., Qin, R., Gao, C.Y., Wu, X.L., Hafezalkotob, A., Herrera, F. (2019), Score-HeDLiSF: A Score Function of Hesitant Fuzzy Linguistic Term Set Based on Hesitant Degrees and Linguistic Scale Functions: An Application to Unbalanced Hesitant Fuzzy Linguistic MULTIMOORA. Information Fusion, 48, 39-54; 
Zhiqiang Liao, Huchang Liao, Xunjie Gou, ZeshuiXu, EdmundasKazimierasZavadskas

[15] Liao, H.C., Xu, Z.S., Herrera-Viedma, E., Herrera, F. (2018), Hesitant Fuzzy Linguistic Term Set and Its Application in Decision Making: A State-of-the Art Survey. International Journal of Fuzzy Systems, 20(7),2084-2110;

[16] Liao, H.C., Xu, Z.S., Zeng, X.J. (2015a), Hesitant Fuzzy Linguistic VIKOR Method and its Application in Qualitative Multiple Criteria Decision Making. IEEE Transactions on Fuzzy Systems, 23, 1343-1355;

[17] Liao, H.C., Xu, Z.S., Zeng, X.J., Merigo, J.M. (2015b), Qualitative Decision Making with Correlation Coefficients of Hesitant Fuzzy Linguistic Term Sets. Knowledge-Based Systems, 76, 127-138;

[18] Rodriguez, R.M., Martinez, L., Herrera, F. (2012), Hesitant Fuzzy Linguistic Term Sets for Decision Making. IEEE Transactions on Fuzzy Systems, 20, 109-119;

[19] Sugeno, M. (1974). Theory of Fuzzy Integrals and its Applications.

$\mathrm{PhD}$ Thesis, Tokyo Institute of Technology, Tokyo;

[20] Wang, X.T., Triantaphyllou, E. (2008), Ranking Irregularities when Evaluating Alternatives by Using Some ELECTRE Methods. Omega, 36, 45-63;

[21] Wu, X.L. and Liao, H.C. (2018), An Approach to Quality Function Deployment Based on Probabilistic Linguistic Term Sets and ORESTE Method for Multi-Expert Multi-Criteria Decision Making. Information Fusion, 43, 13-26;

[22] Wu, X.L. and Liao, H.C. (2019), A Consensus-Based Probabilistic Linguistic Gained and Lost Dominance Score Method. European Journal of Operational Research, 272, 1017-1027;

[23] Wu, X.L., Liao, H.C., Xu Z.S., Hafezalkotob, A., Herrera, F. (2018), Probabilistic Linguistic MULTIMOORA: A Multi-Criteria Decision Making Method Based on the Probabilistic Linguistic Expectation Function and the Improved Borda Rule. IEEE Transactions on Fuzzy Systems, 26, 3688-3702;

[24] Xu, Z.S. (2006), A Note on Linguistic Hybrid Arithmetic Averaging Operator in Multiple Attribute Group Decision Making with Linguistic Information. Group Decision and Negotiation, 15, 593-604;

[25] Zadeh, L.A. (1975), The Concept of a Linguistic Variable and its Application to Approximate Reasoning-I. Information Sciences, 8, 199-249. 